\title{
Supporting Information \\ FRET-based determination of the exchange dynamics of complex coacervate core micelles
}

Inge Bos, Marga Timmerman, and Joris Sprakel *

Physical Chemistry and Soft Matter, Wageningen University \& Research, Stippeneng 4, 6708 WE, Wageningen, The Netherlands

\section{S1 $\quad{ }^{1} \mathbf{H}$ NMR spectra}

${ }^{1} \mathrm{H}$ NMR spectra were recorded on a Bruker Avance $400 \mathrm{MHz}$ NMR equipped with a BBO probe. A $20 \mathrm{kHz}$ spectral width was used. For every measurement the signal from 32 consecutive acquisitions was added to obtain sufficient signal-to-noise ratio. The measured free induction decays were Fourier transformed, phased, baseline corrected and integrated by using the Bruker NMR software TopSpin 4.0.7.

\section{S1.1 Dye to chain transfer agent coupling}

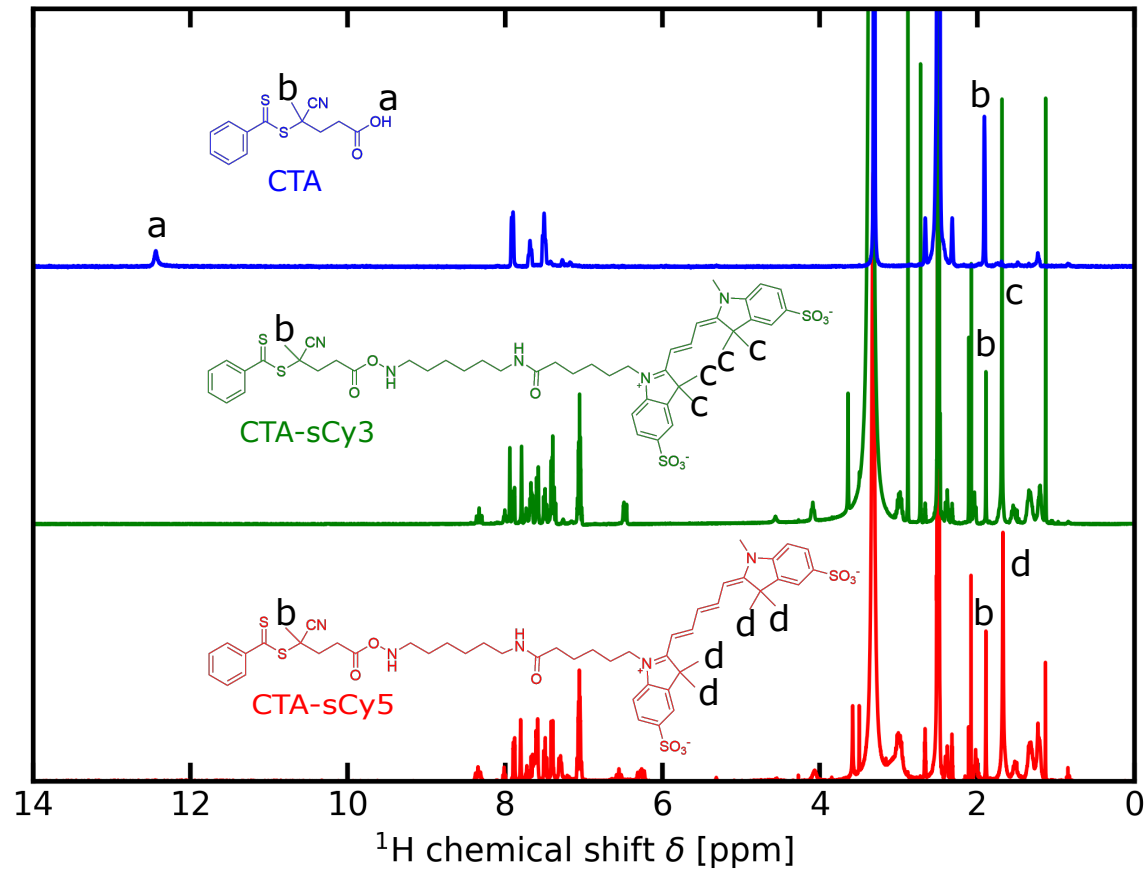

Figure S1. ${ }^{1} \mathrm{H}$ NMR spectra of the chain transfer agent (CTA), the chain transfer agent after coupling to sulfo cyanine3 (CTA-sCy3) and the chain transfer agent after coupling to sulfo cyanine5 (CTA-sCy) in deuterated DMSO. The disappearance of the proton peak $a$ indicates successful removal of unfunctionalised CTA. The $c: b$ ratio in CTA-sCy3 is 15:3 and the $d: b$ ratio in CTA-sCy5 is 13:3. Both are slightly higher than the expected 12:3 ratio, indicating that still some free dye is present. This small dye excess does not react during the polymerisation and is removed in subsequent the dialysis step.

${ }^{*}$ Corresponding author: joris.sprakel@wur.nl 


\section{S1.2 PEG-b-PTMAEMA diblock}

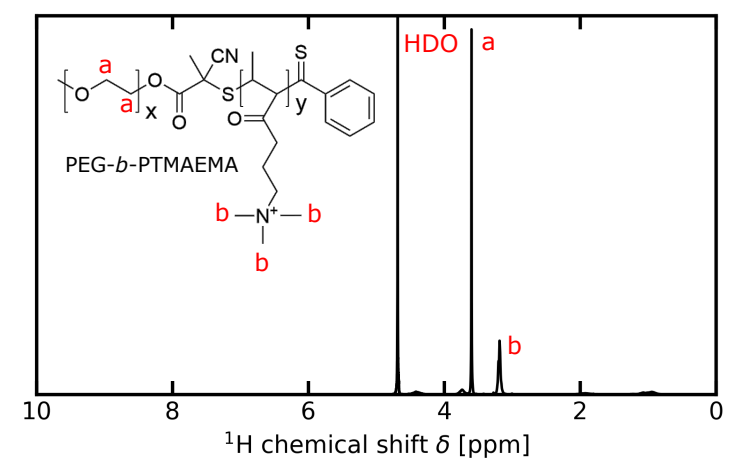

Figure S2. ${ }^{1} \mathrm{H}$ NMR spectrum of the PEG- $b$-PTMAEMA diblock in $\mathrm{D}_{2} \mathrm{O}$. The $a: b$ ratio is 7.2:5.5. The degree of polymerisation of the PEG block is $x=220$. Therefore, the degree of polymerisation of the PTMAEMA block $y \approx 4 x / 9 \cdot 5.5 / 7.2 \approx 75$.

\section{S2 Light scattering experiments}

\section{S2.1 Micelle molar mass estimation}
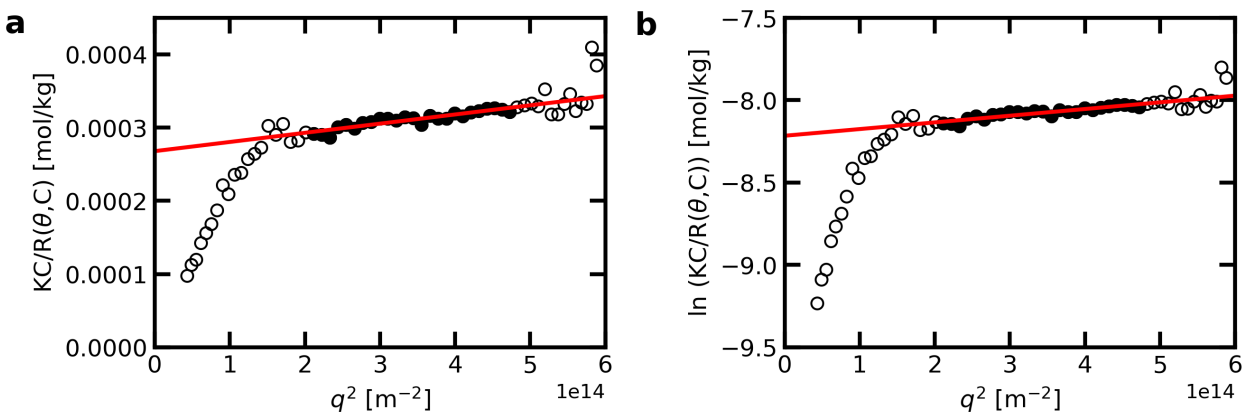

Figure S3. (a) Zimm plot and (b) Guinier plot for light scattering data of donor micelles at a label fraction $\alpha=1.0$ and a $\mathrm{KCl}$ concentration of $100 \mathrm{mM} \mathrm{KCl}$. The red line indicates the linear fit of the data from which the intercept and thus the micelle molar mass was estimated. For this fit, only the data points marked with solid symbols are used.

Table S1. Estimated molar masses $M_{w}$, micelle radius of gyaration $R_{g}$ and average number of donors $N_{D}$ and acceptors $N_{A}$ per micelle based on Zimm and Guinier analysis of light scattering experiments of donor micelles at a label fraction of $\alpha=1.0$ and a SPMA concentration of $1 \mathrm{mM}$ for different $\mathrm{KCl}$ concentrations.

\begin{tabular}{|l|c|c|l|l|l|l|l|l|}
\hline \multirow{2}{*}[\mathbf{KCl}]{$[\mathbf{m M}]$} & \multicolumn{1}{|c|}{$M_{w}[\mathrm{~kg} / \mathbf{m o l}]$} & \multicolumn{2}{c|}{$R_{g}[\mathbf{n m}]$} & \multicolumn{2}{c|}{$N_{D}$} & \multicolumn{2}{c|}{$N_{A}$} \\
\cline { 2 - 9 } & Zimm & Guinier & Zimm & Guinier & Zimm & Guinier & Zimm & Guinier \\
\hline 10 & $5.1 \times 10^{3}$ & $5.1 \times 10^{3}$ & 35 & 33 & 46 & 46 & 76 & 75 \\
100 & $3.7 \times 10^{3}$ & $3.7 \times 10^{3}$ & 37 & 35 & 33 & 33 & 55 & 55 \\
200 & $2.8 \times 10^{3}$ & $2.8 \times 10^{3}$ & 43 & 39 & 25 & 25 & 41 & 41 \\
300 & $3.0 \times 10^{3}$ & $3.0 \times 10^{3}$ & 41 & 37 & 27 & 27 & 45 & 44 \\
400 & $2.7 \times 10^{3}$ & $2.7 \times 10^{3}$ & 42 & 38 & 24 & 24 & 39 & 39 \\
\hline
\end{tabular}




\section{S2.2 Determination of the critical salt concentration}

To determine the critical salt concentration at which the micelles fall apart, we stepwise increased the $\mathrm{KCl}$ concentration and measured the light scattering intensity after every addition step (Fig. S4). When the micelles disassemble, the scattering intensity decreases until they reach the point where all micelles are completely disintegrated. This point corresponds to the critical salt concentrations and above this concentration the light scattering per polymer remains approximately constant. In this case, the critical salt concentration is thus $\sim 790 \mathrm{mM}$. We note that the increase in light scattering intensity around $\sim 670$ $\mathrm{mM}$ is probably the result of micelle rearrangement from spherical to wormlike micelles.[1] The wormlike micelles have a larger aggregation number and therefore scatter more light.

We performed the light scattering titrations on an ALV instrument equipped a $660 \mathrm{~nm}$ laser at a $90^{\circ}$ detection angle. A Schott-Geräte computer-controlled titration setup was used to regulate the addition of titrant, stirring, and delay time between additions. A $6 \mathrm{~mL}$ solution of donor micelles $([\mathrm{SPMA}]=1$ $\mathrm{mM}$ ) in $10 \mathrm{mMKCl}$ was prepared. To this solution, a stock solution of $2 \mathrm{M} \mathrm{KCl}$ was added in steps of $0.025 \mathrm{~mL}$. After a stirring and delay time of both $60 \mathrm{~s}$, the light scattering intensity was recorded in 10 independent runs of $20 \mathrm{~s}$. The recorded light scattering intensities $I$ are corrected for the total polymer weight concentration $C$.

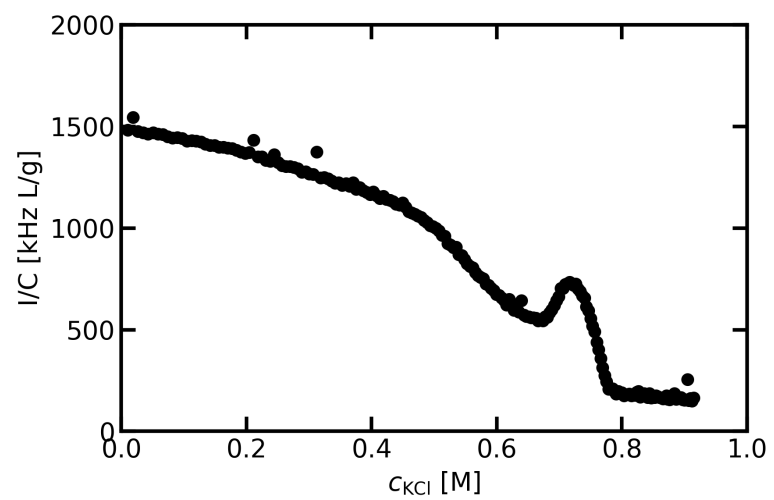

Figure S4. Light scattering salt titrations of donor micelles with a label fraction $\alpha=1.0$ starting at a SPMA concentration of $1 \mathrm{mM}$.

\section{S3 Deconvolution of the emission spectrum}

To determine the relative contributions of the donor emission and acceptor emission to the total emission spectrum, we have fit the total spectrum with a weighted sum of the separate donor and acceptor spectra:

$$
I_{\text {tot }}(\lambda)=d I_{D}(\lambda)+a I_{A}(\lambda)
$$

Here $I_{\text {tot }}(\lambda)$ it the total intensity at a wavelength $\lambda$ and $I_{D}(\lambda)$ and $I_{A}(\lambda)$ are the intensity of the donor and acceptor spectrum at this wavelength respectively. The prefactors $d$ and $a$ give the relative contribution of the donor and acceptor. These prefactors are the fit parameters. To perform this fit, we have first approximated both the donor and acceptor emission spectrum with a sum of a two log normal functions:

$$
I(\lambda)=\frac{x}{\lambda \sigma_{1} \sqrt{2 \pi}} \exp \left(-\frac{\left(\ln (\lambda)-\mu_{1}\right)^{2}}{2 \sigma_{1}^{2}}\right)+\frac{1-x}{\lambda \sigma_{2} \sqrt{2 \pi}} \exp \left(-\frac{\left(\ln (\lambda)-\mu_{2}\right)^{2}}{2 \sigma_{2}^{2}}\right)
$$

here $I(\lambda)$ is the intensity of the donor or acceptor. For the donor spectrum, we have used $x=0.535$, $\sigma_{1}=1.90 \times 10^{-2}, \mu_{1}=6.34, \sigma_{2}=3.99 \times 10^{-2}$ and $\mu_{2}=6.39$. For the acceptor spectrum, we have used $x=0.678, \sigma_{1}=2.16 \times 10^{-2}, \mu_{1}=6.51, \sigma_{2}=5.29 \times 10^{-2}$ and $\mu_{2}=6.55$. These values were obtained by fitting Equation S2 to the donor or acceptor emission spectrum using a Monte Carlo fit algorithm.

To fit Equation S1 to the measured emission spectra, we have used the non-linear least square fit algorithm as implemented in the python package SciPy. Two fit examples are given in Fig. S5. After fitting the 
data, the total donor intensity and total acceptor intensity, needed to calculate the FRET efficiency, were obtained by integrating $d I_{D}(\lambda)$ and $a I_{A}(\lambda)$ over the wavelength range $\lambda=500 \mathrm{~nm}$ to $\lambda=800 \mathrm{~nm}$.
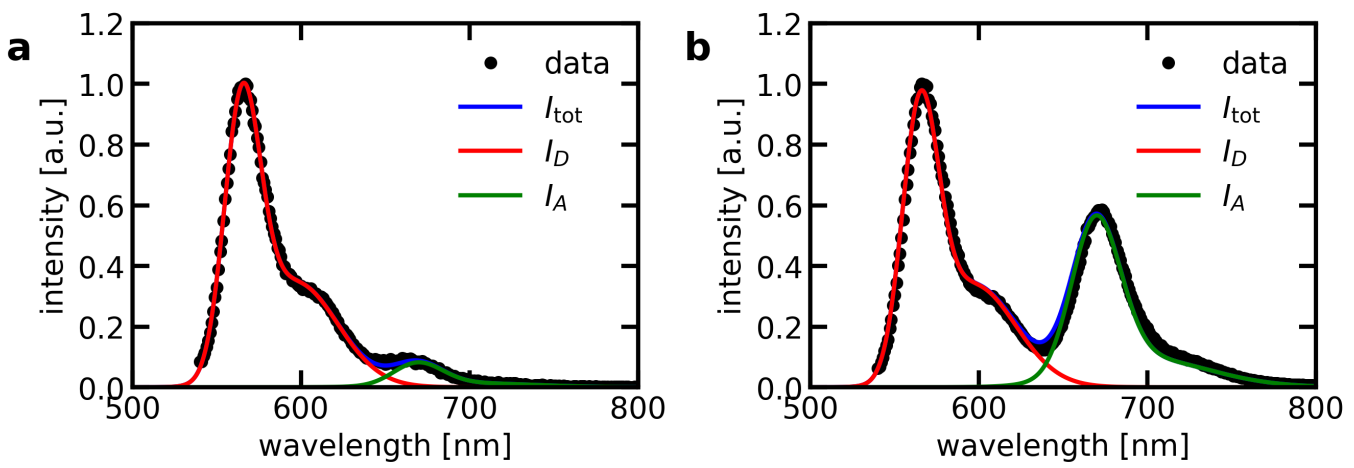

Figure S5. Fit of Equation S1 to two example emission spectra corrected for direct acceptor excitation. (a) Emission spectrum measured in an exchange measurement at $100 \mathrm{mM} \mathrm{KCl,} \mathrm{a} \mathrm{SPMA} \mathrm{concentration} \mathrm{of} 1 \mathrm{mM}$, a label fraction $\alpha=0.2$ at $t=4.0 \mathrm{~h}$ (b) Emission spectrum of mixed micelles at $100 \mathrm{mM} \mathrm{KCl}$, a SPMA concentration of $1 \mathrm{mM}$ and a label fraction $\alpha=1.0$.

\section{S4 FRET intensity $E(t)$ as function of time after mixing $t$}
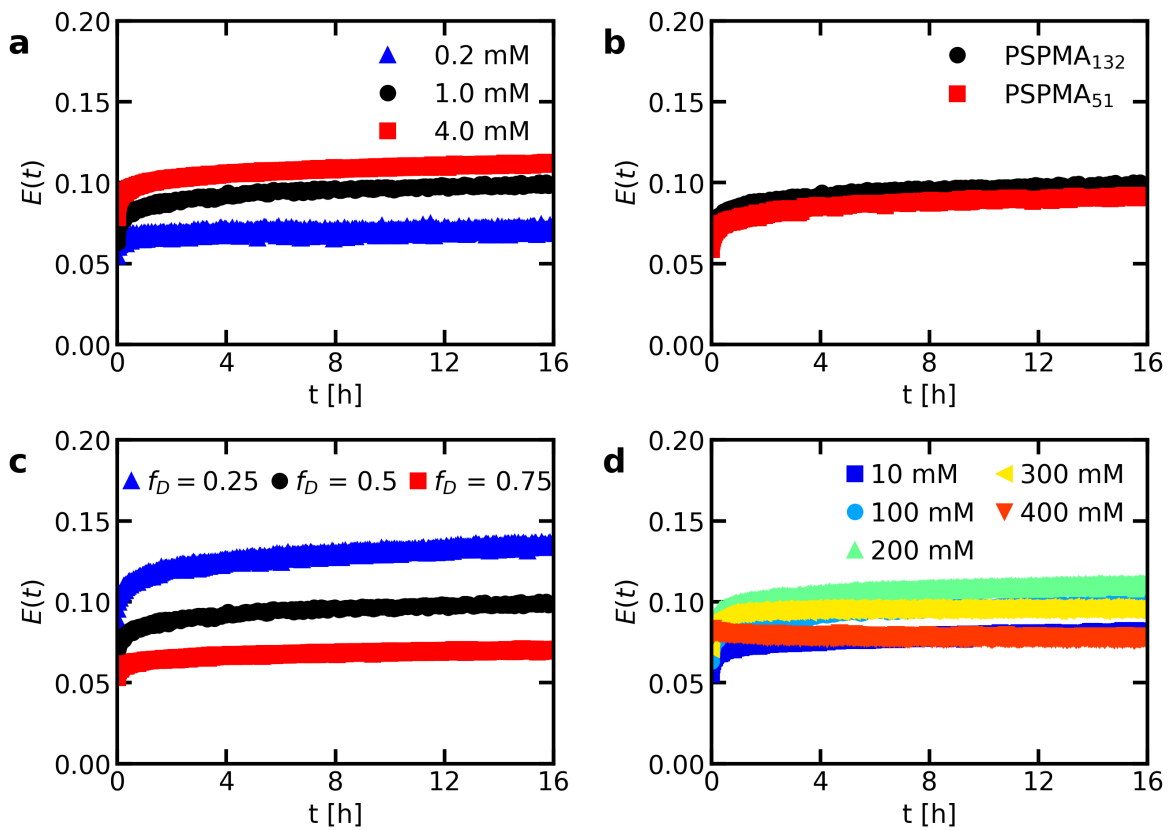

Figure S6. FRET efficiency $E(t)$ as function of time after mixing $t$ for (a) different monomer concentrations; (b) different unlabelled homopolymer lengths; (c) different fractions of donor micelles and (d) different salt concentrations. 


\section{S5 Estimation of the free polymer fraction}

For all micelle concentrations, the concentration of polymers that are not part of a micelle (the free polymers) is equal to the critical micelle concentration $\mathrm{cmc}$. The free polymer fraction $f$ at a concentration $c$ is thus given by:

$$
f= \begin{cases}1, & \text { if } c \leq c m c \\ \frac{c m c}{c}, & \text { if } c>c m c\end{cases}
$$

Only the polymers that are part of the micelle are close enough to each other for FRET to occur and therefore only these polymers contribute to the FRET efficiency of the sample. The measured FRET efficiency will thus depend on the free polymer fraction, which decreases with increasing monomer concentration (Equation S3). We have measured an apparent FRET efficiency $E^{\text {app }}$ at different monomer concentrations (Fig. S4). We call it an apparent FRET efficiency because we did not correct for direct acceptor excitation and differences in self-quenching. This apparent FRET efficiency can be related to the free polymer fraction by:

$$
E^{\text {app }}=(1-f) E_{\text {micelle }}+E_{\text {baseline }}
$$

here $(1-f)$ is the fraction of polymers that are part of a micelle, $E_{\text {micelle }}$ is the average FRET efficiency in a micelle (without correction for differences in self-quenching) and $E_{\text {baseline }}$ is a correction term to account for direct acceptor excitation. We note that taking $E_{\text {baseline }}$ as a constant is a simplification, as in reality the direct acceptor excitation emission will depend on whether the acceptor polymers are part of a micelle or not due to refractive index differences and differences in self-quenching. However, we do not expect that this simplification highly affects the onset of the FRET increase and thus also hardly has an effect the determination of the critical micelle concentration.

By comparing Equations S1 and S4 to the epxerimental data, we find that for $10 \mathrm{mM} \mathrm{KCl} \mathrm{the} \mathrm{critical}$ micelle concentration is $\sim 7 \mu \mathrm{M}$ and for $400 \mathrm{mM} \mathrm{KCl}$ the critical micelle concentration is $\sim 20 \mu \mathrm{M}$ (Fig. $\mathrm{S} 7$ ). For micelles at $400 \mathrm{mM} \mathrm{KCl}$ and a SPMA monomer concentration $c=1 \mathrm{mM}$, the free polymer fraction is thus $f \approx 0.02$.

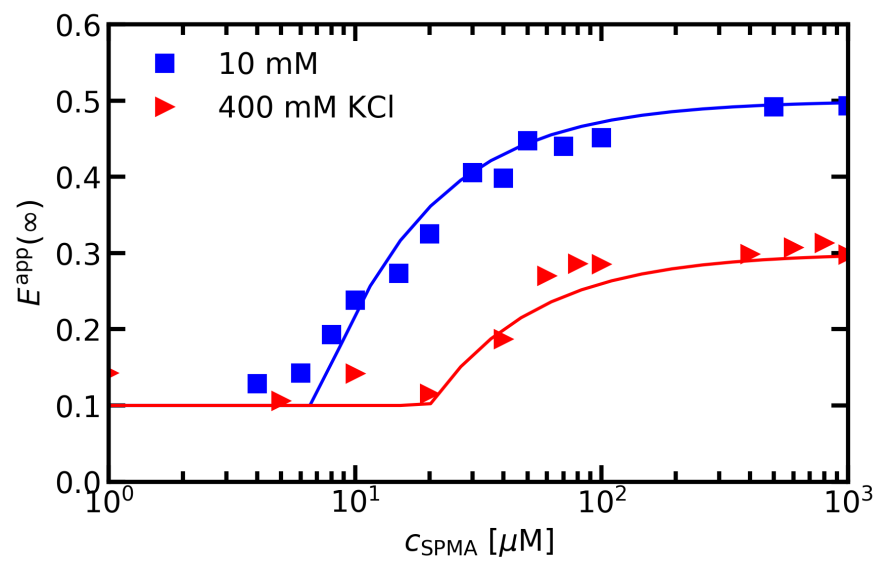

Figure S7. Apparent FRET efficiency $E^{\mathrm{app}}(\infty)$ of mixed micelles at a label fraction $\alpha=1.0$ as function of SPMA monomer concentration. This apparent FRET efficiency is not corrected for direct acceptor excitation and differences between donor and acceptor self-quenching. Solid lines are calculated with Equations S3 and S4 with $\mathrm{cmc}=7 \mu \mathrm{M}, E_{\text {micelle }}=0.40$ and $E_{\text {baseline }}=0.10$ for $10 \mathrm{mM} \mathrm{KCl}$ and $\mathrm{cmc}=20 \mu \mathrm{M}, E_{\text {micelle }}=0.20$ and $E_{\text {baseline }}=0.10$ for $400 \mathrm{mM} \mathrm{KCl}$. 


\section{S6 Bleaching test of equilibrated mixed micelles}

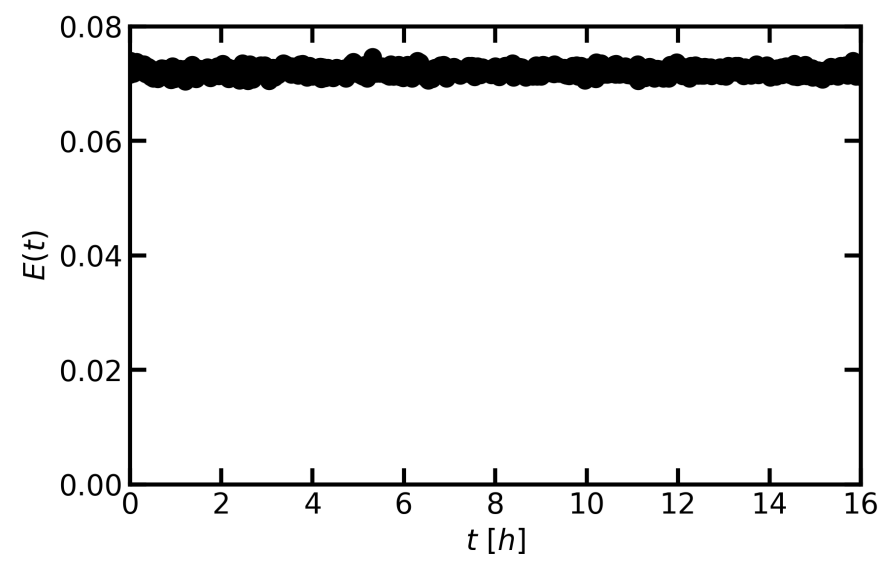

Figure S8. FRET efficiency $E$ of mixed micelles at a label fraction $\alpha=0.2$, a $400 \mathrm{mM} \mathrm{KCl}$ concentration and a SPMA concentration of $1.0 \mathrm{mM}$ measured over $16 \mathrm{~h}$.

\section{S7 Fits of the activated process models to the FRET micelle exchange data at different salt concentrations}
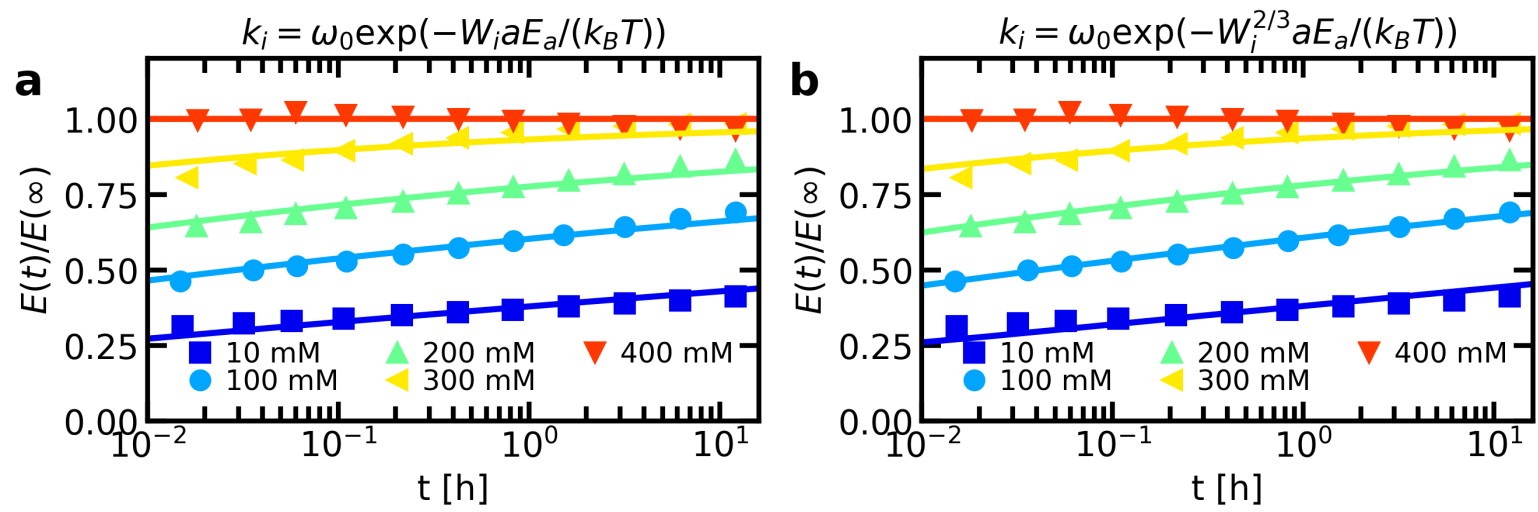

Figure S9. Fits of the activated process models to the FRET micelle exchange data at different salt concentrations using $\omega_{0}$ as shared fit parameter. To ensure that the data at short and long time scales have similar weighting, the exchange data is first binned in time steps that are evenly spaced on a logarithmic time scale. The values for $\nu, N$ and the polymer length distributions are used as constants in the fit calculation. The values for $\nu$ and $N$ are obtained from Table 1 of the main text and the polymer length distributions are obtained from GPC data assuming a Schulz-Zimm distribution. (a) Fit to an activated process where the total activation energy scales linearly with the polymer length $\left(k_{i}=\omega_{0} \exp \left(-W_{i} E_{a} /\left(k_{B} T\right)\right)\right.$ ) yielding $\omega_{0}=9.5 \times 10^{2} \mathrm{~Hz}$ and $a E_{a}=0.54 k_{B} T, a E_{a}=0.32 k_{B} T, a E_{a}=0.22 k_{B} T, a E_{a}=0.14 k_{B} T$ and $a E_{a}=0.04 k_{B} T$ for a salt concentration of $10 \mathrm{mM}, 100 \mathrm{mM}, 200 \mathrm{mM}, 300 \mathrm{mM}$ and $400 \mathrm{mM}$ respectively. (b) Fit to an activated process where the total activation energy scales with the $2 / 3$ power the polymer length $\left(k_{i}=\omega_{0} \exp \left(-W_{i}^{2 / 3} E_{a} /\left(k_{B} T\right)\right)\right.$ yielding $\omega_{0}=1.2 \times 10^{6} \mathrm{~Hz}$ and $a E_{a}=2.4 k_{B} T, a E_{a}=1.7 k_{B} T, a E_{a}=1.3 k_{B} T, a E_{a}=0.97 k_{B} T$ and $a E_{a}=0.42 k_{B} T$ for a salt concentration of $10 \mathrm{mM}, 100 \mathrm{mM}, 200 \mathrm{mM}, 300 \mathrm{mM}$ and $400 \mathrm{mM}$ respectively. 


\section{S8 Analytical solution of the differential equation for $P(n, t)$}

As explained in the main text, the differential equation describing the changes in probability $P$ to find a micelle with $n$ fluorophores is given by:

$$
\frac{d P(n, t)}{d t}=f N k P(n-1, t)-(f N k+n k) P(n, t)+(n+1) k P(n+1)
$$

By using the generating function $G(s, t)=\sum_{n=0}^{\infty} s^{n} P(n, t)$ we can rewrite Equation S5 to:

$$
\frac{\partial G(s, t)}{\partial t}=\sum_{n=0}^{\infty} s^{n}[f N k P(n-1, t)-(f N k+n k) P(n, t)+(n+1) k P(n+1)]
$$

A change of summation variables $n \rightarrow n-1$ and $n \rightarrow n+1$ in the first and last term respectively gives:

$$
\begin{aligned}
\frac{\partial G(s, t)}{\partial t} & =\sum_{n=0}^{\infty} s^{n+1} f N k P(n, t)-s^{n}(f N k+n k) P(n, t)+s^{n-1} n k P(n, t) \\
& =\sum_{n=0}^{\infty} f N k\left(s^{n+1}-s^{n}\right) P(n, t)+n k\left(s^{n-1}-s^{n}\right) P(n, t) \\
& =(s-1) f N k \sum_{n=0}^{\infty} s^{n} P(n, t)+k\left(s^{-1}-1\right) \sum_{n=0}^{\infty} s^{n} n P(n, t)
\end{aligned}
$$

The $\sum_{n=0}^{\infty} s^{n} n P(n, t)$ part in Equation $\mathrm{S} 7$ can be rewritten by making use of the property that $J(a, x)=$ $\sum_{n} a x^{n}$ gives $x \frac{\partial J}{\partial x}=\sum_{n} a n x^{n}:$

$$
\begin{aligned}
\frac{\partial G(s, t)}{\partial t} & =(s-1) f N k \sum_{n=0}^{\infty} s^{n} P(n, t)+k\left(s^{-1}-1\right) s \frac{\partial}{\partial s} \sum_{n=0}^{\infty} s^{n} P(n, t) \\
& =(s-1) f N k G(s, t)-k(s-1) \frac{\partial G(s, t)}{\partial s}
\end{aligned}
$$

The steady state solution of this partial differential equation at $t \rightarrow \infty$ follows from $\frac{\partial G_{s t}}{\partial t}=0$ and $G(1, t)=1$ :

$$
G_{s t}=e^{f N(s-1)}=e^{-f N} e^{f N s}=e^{-f N} \sum_{n=0}^{\infty} \frac{(f N s)^{n}}{n !}=\sum_{n=0}^{\infty} s^{n} e^{-f N} \frac{(f N)^{n}}{n !}=\sum_{n=0}^{\infty} s^{n} P_{s t}(n)
$$

The steady state probability distribution $P_{s t}$ is thus given by

$$
P_{s t}(n)=e^{-f N} \frac{(f N)^{n}}{n !}
$$

This is a Poisson distribution with an average of $f N$.

The time-dependent solution of the partial differential equation (Equation S8) can be found by using the Lagrange method. This gives as general solution:

$$
G e^{-f N s}=F\left(s-1 e^{-k t}\right)
$$

where $F$ is any analytical function. The specific solution can be found from the initial condition. We distinguish two cases:

1. The number of donors in acceptor micelles and vice versa

2. The number of donors in donor micelles and the number of acceptors in acceptor micelles 
In the first case, the initial fluorophore number is 0 , therefore $P(n=0,0)=1$ and $P(n \neq 0,0)=0$. This gives $G(s, 0)=1$. From this initial condition we get for the first case:

$$
F\left((s-1) e^{-k t}\right)=e^{-f N} e^{-f N(s-1) e^{-k t}}
$$

Substitution of Equation S12 in Equation S11 gives the specific solution for the first case:

$$
G(s, t)=e^{\lambda(t)(s-1)}
$$

where $\lambda(t)=f N\left(1-e^{-k t}\right)$. This can be rewritten to:

$$
G(s, t)=e^{-\lambda(t)} \sum_{n=0}^{\infty} \frac{(\lambda(t) s)^{n}}{n !}=\sum_{n=0}^{\infty} s^{n} e^{-\lambda(t)} \frac{(\lambda(t))^{n}}{n !}=\sum_{n=0}^{\infty} s^{n} P(n, t)
$$

The probability $P$ at a certain time $t$ to have a donor micelle with $n$ acceptors or an acceptor micelle with $n$ donors is thus given by:

$$
P(n, t)=e^{-\lambda(t)} \frac{\lambda(t)^{n}}{n !}
$$

For the second case we make use of the fact that the donor micelles and acceptor micelles are equilibrated when they are mixed. This means that they are in steady state. Only in this case, no other micelle types are present and therefore $f=1$. Their initial distribution is thus given by: $P(n, 0)=e^{-N} \frac{N^{n}}{n !}$. This gives $G(s, 0)=e^{-N} \sum_{n=0}^{\infty} \frac{(s N)^{n}}{n !}=e^{N(s-1)}$. In this way we get for the second case:

$$
F\left((s-1) e^{-k t}\right)=e^{-f N} e^{(N-f N)(s-1) e^{-k t}}
$$

Substitution of Equation S16 in Equation S11 gives the specific solution for the second case:

$$
G(s, t)=e^{(s-1) \mu(t)}
$$

where $\mu(t)=N\left(f+(1-f) e^{-k t}\right)$. Equation S17 can again be rewritten to give the probability to find a donor micelle with $n$ donors or an acceptor micelle with $n$ acceptors at a time $t$ :

$$
P(n, t)=e^{-\mu(t)} \frac{\mu(t)^{n}}{n !}
$$

\section{References}

(1) Van der Kooij, H. M.; Spruijt, E.; Voets, I. K.; Fokkink, R.; Cohen Stuart, M. A.; van der Gucht, J. On the stability and morphology of complex coacervate core micelles: From spherical to wormlike micelles. Langmuir 2012, 28, 14180-14191. 